DIW BERLIN

Discussion

Papers
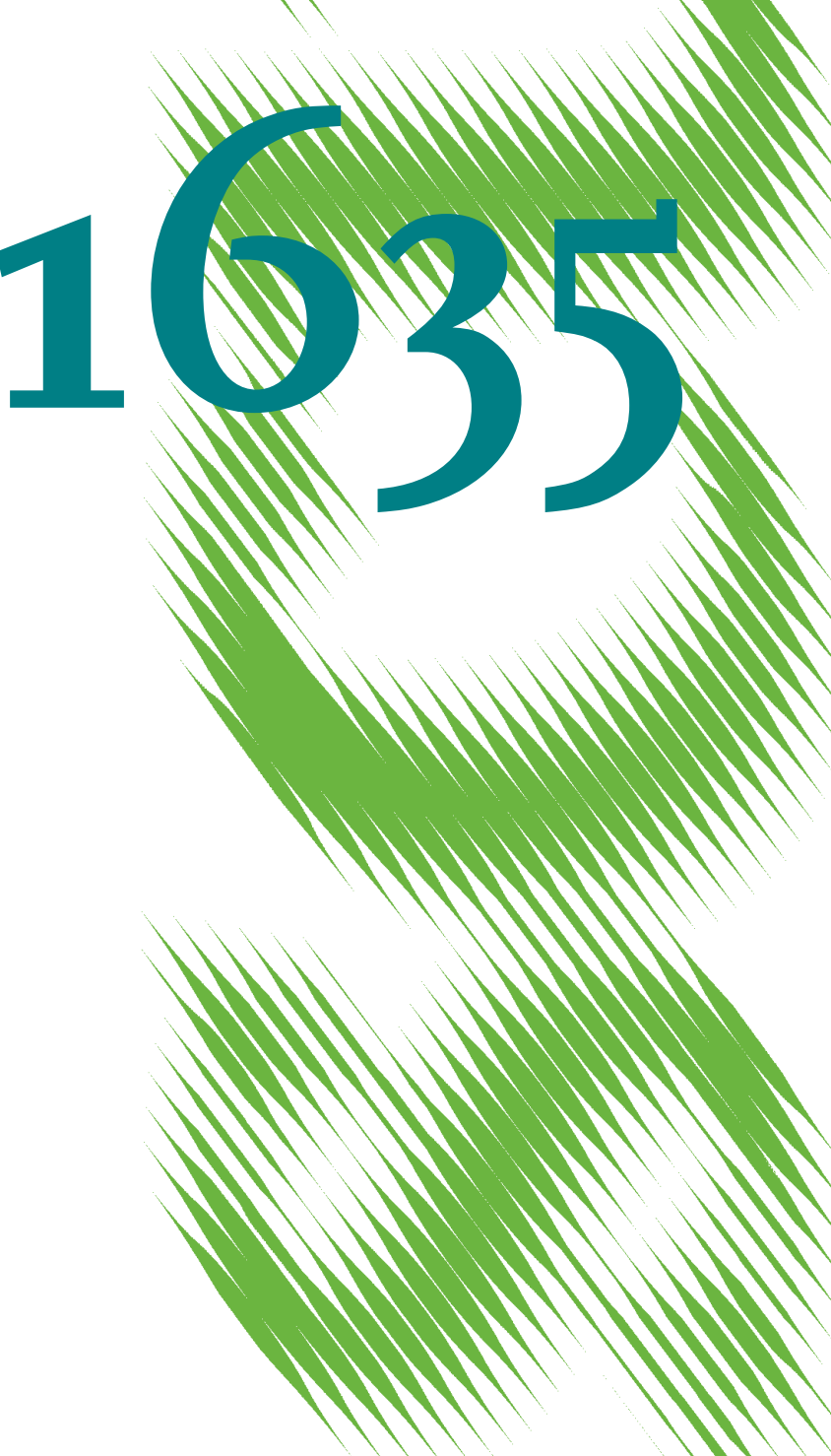

MIMMMMMMMIIII

Upstream Monopoly and

Downstream Information Sharing 
Opinions expressed in this paper are those of the author(s) and do not necessarily reflect views of the institute.

IMPRESSUM

(C) DIW Berlin, 2017

DIW Berlin

German Institute for Economic Research

Mohrenstr. 58

10117 Berlin

Tel. +49 (30) $89789-0$

Fax +49 (30) $89789-200$

http://www.diw.de

ISSN electronic edition 1619-4535

Papers can be downloaded free of charge from the DIW Berlin website:

http://www.diw.de/discussionpapers

Discussion Papers of DIW Berlin are indexed in RePEc and SSRN:

http://ideas.repec.org/s/diw/diwwpp.html

http://www.ssrn.com/link/DIW-Berlin-German-Inst-Econ-Res.html 


\title{
Upstream Monopoly and Downstream Information Sharing
}

\author{
Pio Baake* Andreas Harasser ${ }^{\dagger}$ \\ DIW Berlin DIW Berlin
}

January 31, 2017

\begin{abstract}
We analyze a vertical structure with an upstream monopoly and two downstream retailers. Demand is uncertain but each retailer receives an informative private signal about the state of the demand. We construct an incentive compatible and ex ante balanced mechanism which induces the retailers to share their information truthfully. Information sharing can be profitable for the retailers but is likely to be detrimental for social welfare.
\end{abstract}

JEL Classifications D82, L13, L14

Keywords information sharing, upstream monopoly, vertical relations

\section{Introduction}

Uncertainty of demand is a problem for firms in almost any type of industry. In this model we analyze a vertical structure with an upstream monopoly and two downstream retailers. Demand is uncertain but each retailer receives an informative private signal about the state of the demand. The upstream monopoly can offer incentive compatible take-it-orleave-it contracts to the retailers. Allowing for information sharing between the retailers, we construct an incentive compatible and ex ante balanced mechanism which induces the retailers to share their information truthfully. We show that while information sharing can be profitable for the retailers, it is likely to be detrimental for social welfare as the

*DIW Berlin, Mohrenstr. 58, 10117 Berlin, Germany, Email: pbaake@diw.de.

${ }^{\dagger}$ DIW Berlin, Mohrenstr. 58, 10117 Berlin, Germany, Email: aharasser@diw.de. 
contracts offered by the upstream monopoly tend to involve more downward distortion with information sharing.

There is a number of papers, which analyze firms' incentives to share information in a single tier industry with uncertain demand. Clark (1983) shows that firms only have an incentive to share their private information industry wide, if they are able to enforce a collusive agreement vis-à-vis consumers. Gal-Or (1985) analyzes a situation, where firms receive correlated private signals about the state of demand. However, it is shown that firms do not want to share their information regardless of the degree of correlation of the signals. Vives (1984) shows for a duopoly market with substitute goods that firms want to share information under Bertrand, while they do not want to share under Cournot competition, with results being reversed if the firms' goods are complements. Vives (1990) on the other hand analyzes the effects of different information disclosure rules on profits and welfare in a market with monopolistic competition. He finds that only exclusionary disclosure, meaning that only firms participating in the sharing mechanism receive the aggregated information, leads firms to share information, while information sharing is only welfare improving in Cournot competition but not in Bertrand competition. ${ }^{1}$ Wagenhofer (1990) takes a similar approach to ours in that he allows a firm to choose a disclosure rule itself, leading to full, partial or no disclosure at all. But while in his paper only one of the firms is informed and chooses a disclosure rule, in our model both downstream firms have private information and have to agree on a disclosure rule.

In line with Ziv (1993), we introduce an ex ante balanced, truthfully revealing mechanism for information sharing. While in his paper there is only one industry layer, we introduce a supplier, who - by using incentive compatible contracts - gives the firms an incentive to share information in the first place.

Jiang and Hao (2016) also analyze a vertical structure and allow for both horizontal information sharing and information acquisition by the supplier from retailers. However, while their paper focuses on the determinants of information flow within such an industry, our paper concentrates on the retailers' incentives to share their information truthfully. Similar to the literature on countervailing power (see for instance Dobson and Waterson,

\footnotetext{
${ }^{1}$ A general model of horizontal information acquisition, which also presents an overview of the hitherto existing literature, is presented by Raith (1996).
} 
1997, for a theoretical analysis or Ellison and Snyder, 2010, for an empirical evaluation of the argument of countervailing power), our paper analyzes downstream firms' incentives to cooperate, when facing a monopoly supplier, and the impact of the firms' decisions concerning cooperation on welfare. As in buyer groups in our model the retailers are able to improve their position vis-à-vis the supplier, as information sharing allows the retailers to better predict the state of demand and makes them less prone to the supplier's price discrimination. However, in contrast to this literature, the firms in our model cannot use cooperation to exercise any bargaining power directly, but only via the improvement of their information.

Our contribution to the aforementioned literature is the analysis of horizontal information sharing and its impact on contract offers of an upstream monopolist. Furthermore, we focus on the incentives for downstream firms to share information and allow them to agree on having either no, partial or full information sharing. We also show the existence of an ex ante balanced mechanism to ensure truthful information sharing. In our model, welfare effects are ambiguous. If the precision of the retailers' signals is sufficiently high, they share more information than socially desirable.

In the following section we set up our model. The game without information sharing is analyzed in section three. In section four we characterize the equilibrium contracts under different patterns of information sharing. A payment scheme which induces the retailers to truthfully reveal their signals is presented in section five. Optimal information sharing is considered in section six. The final section concludes.

\section{The Model}

We consider a simple vertical structure with one upstream supplier and two downstream retailers $i, j \in I=\{1,2\}, i \neq j$. The supplier produces only one good and offers each retailer a menu of take it or leave contracts. These offers are secret and we assume that retailers have passive beliefs. Furthermore, demand is uncertain but before choosing its contract each retailer receives a private and informative signal about the state of the demand. Considering information sharing we allow the retailers to agree on mediated information sharing involving a third party which transforms private messages received 
from the retailers into a common message sent back to them.

The timing and formal structure of our model is the following:

1. State of demand is determined by nature: The inverse demand $P(\alpha, x)$ function can be either high or low

$P(\alpha, x)=a-x$ with $a \in\left\{a_{L}, a_{H}\right\}$ and $\rho_{H}=\operatorname{Pr}\left\{a=a_{H}\right\}=\frac{1}{2}, \rho_{L}=\operatorname{Pr}\left\{a=a_{L}\right\}=\frac{1}{2}$

where $x$ denote the total quantity offered. For simplicity we use $a_{H}=1$ and $a_{L}=$ $\alpha \in[0.5,1]$. While $a_{H}=1$ and $a_{L}$ are common knowledge, the realization of $a$ is observed neither by the supplier nor by the retailers.

2. Retailers decide whether to sign an information sharing agreement. If an agreement is reached it establishes a third party and a rule how the third party transforms messages sent by the retailers into a common message sent back to the retailers. The agreement can also entail payments by the retailers to the third party. Furthermore, the agreement is binding and publicly observable.

3. Each retailer $i$ receives a private signal $s^{i} \in\left\{s_{L}, s_{H}\right\}$ with the following probabilities

$$
\rho_{H H}=\operatorname{Pr}\left\{s^{i}=s_{H} \mid 1\right\}=(1-\xi) \frac{1}{2}+\xi, \rho_{H L}=\operatorname{Pr}\left\{s^{i}=s_{H} \mid \alpha\right\}=(1-\xi) \frac{1}{2}
$$

where $\xi \in[0,1]$ measures the precision of the signals. Note that with $\xi=0$ the retailers' signals are not informative while $\xi=1$ leads to perfect information.

4. If an information sharing agreement was signed retailers decide which message $\widetilde{s}^{i} \in$ $\left\{s_{L}, s_{H}\right\}$ they send to the third party. The third party transforms the signals into a common message $M\left(\widetilde{s}^{i}, \widetilde{s}^{j}\right)$ sent back to the retailers. The message is not observed by the supplier.

We distinguish between complete and partial information sharing. With complete information sharing the third party simply sends both retailers the signals it received:

$$
M^{C}\left(\widetilde{s}^{i}, \widetilde{s}^{j}\right)=\left(\widetilde{s}^{i}, \widetilde{s}^{j}\right)
$$

Considering partial information sharing we focus on $M\left(\widetilde{s}^{i}, \widetilde{s}^{j}\right)$ based on pure strate- 
gies and the following two schemes: Partial information sharing $L$ leads to

$$
M^{P L}\left(\widetilde{s}^{i}, \widetilde{s}^{j}\right)= \begin{cases}\left(s_{L}, s_{L}\right) & \text { if } \quad \widetilde{s}^{i}=\widetilde{s}^{j}=s_{L} \\ (0) & \text { else }\end{cases}
$$

While partial information sharing $H$ implies

$$
M^{P H}\left(\widetilde{s}^{i}, \widetilde{s}^{j}\right)= \begin{cases}\left(s_{H}, s_{H}\right) & \text { if } \quad \widetilde{s}^{i}=\widetilde{s}^{j}=s_{H} \\ (0) & \text { else }\end{cases}
$$

Note that $M^{P L}\left(\widetilde{s}^{i}, \widetilde{s}^{j}\right)$ implies that retailers with signals $s_{L}$ can perfectly infer the other retailer's signal from the message. $M^{P H}\left(\widetilde{s}^{i}, \widetilde{s}^{j}\right)$ leads to the same inference for each retailer who gets signal $s_{H}$.

5. The supplier offers each retailer $i$ a menu of contracts including a fixed transfer payment $T$ and a quantity $x$ based on the information the retailers may have

$$
\begin{gathered}
C_{L}^{i}=\left(T_{L}^{i}, x_{L}^{i}\right), C_{H}^{i}=\left(T_{H}^{i}, x_{H}^{i}\right) \text { without information sharing } \\
C_{L \phi}^{i}=\left(T_{L \phi}^{i}, x_{L \phi}^{i}\right), C_{H \phi}^{i}=\left(T_{H}^{i}, x_{H \phi}^{i}\right) \text { with information sharing }
\end{gathered}
$$

where $\phi$ denotes the inference a retailer can make about the other retailer's signal, given the chosen information sharing regime and the received message. Contract offers are secret and not observed by the other retailer.

6. Retailers decide which contract they choose

7. Quantities are offered on the market and all profits are realized

Figure 1 illustrates the information sets of retailer 1 under the different information sharing agreements (we slightly simplified the notation by using $H$ and $L$ instead of $s_{H}$ and $s_{L}$, respectively). ${ }^{2}$

With partial information sharing the retailers' ex post beliefs depend on their own signal as well as on the inference they can make from the message sent by the third party. Assume

\footnotetext{
${ }^{2}$ We do not consider the case of partial information sharing with different signals (one retailer receiving an $H$ and one receiving an $L$ signal) as this allows full inference of the other retailer's signal and therefore does not differ from complete information sharing.
} 


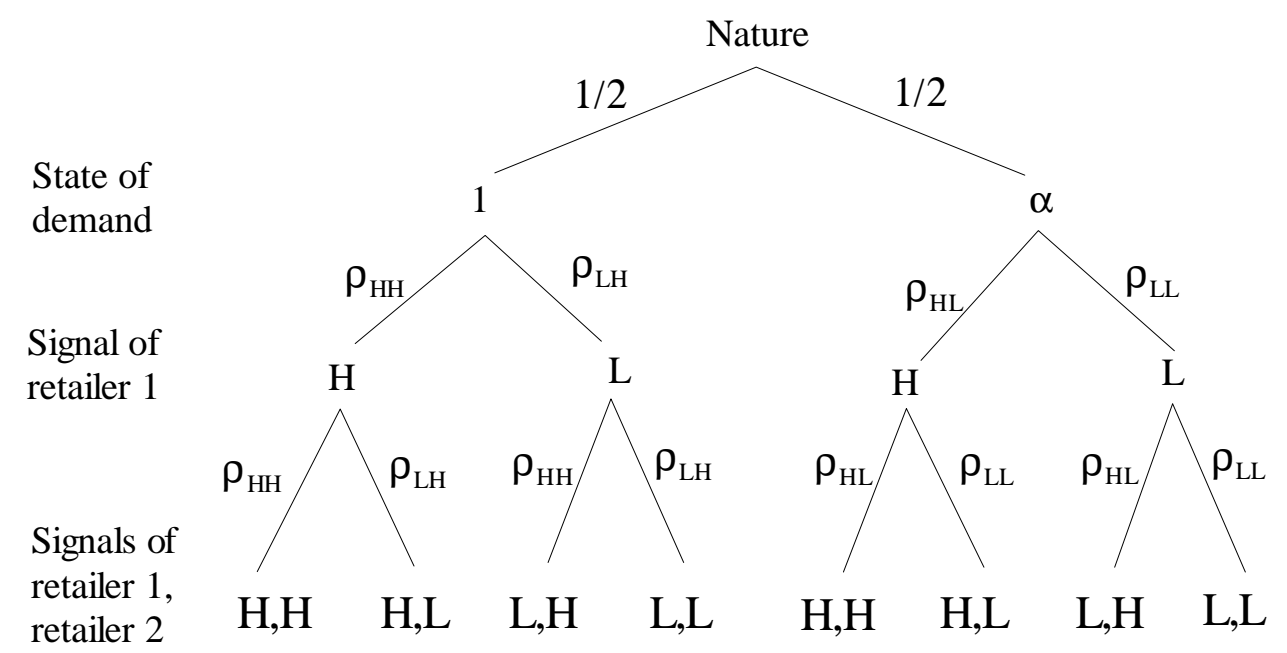

Information of retailer 1 about signals without information sharing

\begin{tabular}{llllllll}
$\mathrm{H}, \mathrm{H}$ & $\mathrm{H}, \mathrm{L}$ & $\mathrm{L}, \mathrm{H}$ & $\mathrm{L}, \mathrm{L}$ & $\mathrm{H}, \mathrm{H}$ & $\mathrm{H}, \mathrm{L}$ & $\mathrm{L}, \mathrm{H}$ & $\mathrm{L}, \mathrm{L}$ \\
\hline
\end{tabular}

Information of retailer 1 about signals with partial information sharing L

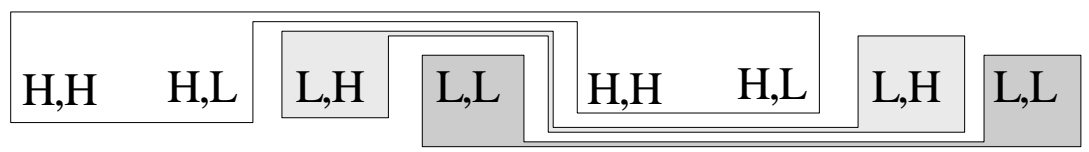

Information of retailer 1 about signals with partial information sharing $\mathrm{H}$

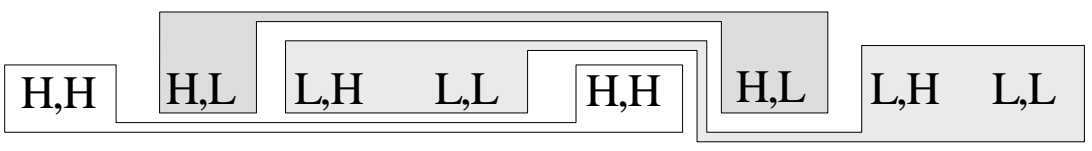

Information of retailer 1 about signals with complete information sharing

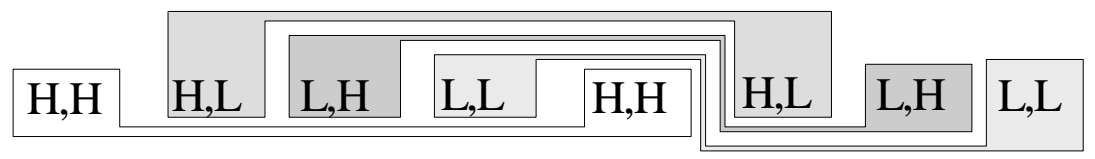

Figure 1: Information sharing and Information sets 
$M^{P L}=\left(s_{L}, s_{L}\right)$ and truthful reports of the signals, ${ }^{3}$ then the retailers know that both received an $L$ signal, which corresponds to the case of $M^{C}=\left(s_{L}, s_{L}\right)$. If a retailer's signal is $s_{L}$ and $M^{P L}=(0)$, he can infer that the other retailer received a signal $s_{H}$. However, the retailer with signal $s_{H}$ receives message $M^{P L}=(0)$ as well, but can not make any inference about the signal of the other retailer, as given $\widetilde{s}^{i}=s_{H}, M^{P L}$ is independent of $\widetilde{s}^{j}$. Hence, there are only three possible signal and information combinations and the supplier can restrict its offers to three possible contracts $C_{L L}^{i}, C_{L H}^{i}$ and $C_{H 0}^{i}$ (see Figure 1). The case with $M^{P H}=\left(s_{H}, s_{H}\right)$ corresponds to the case $M^{C}=\left(s_{H}, s_{H}\right)$. Similarly, if one retailers got the signal $s_{H}$ but receives a message $M=(0)$, he can perfectly infer that the other retailer's signal was $s_{L}$. However, if a retailer's signal is $s_{L}$ he will get message $M=(0)$ irrespectively of the other retailer's signal. Hence, in this case there are again only three possible signal and information combinations leading to three possible contracts $C_{H H}^{i}, C_{H L}^{i}$ and $C_{L 0}^{i}$.

Complete information sharing leads to the case in which each retailer has four possible information sets. From the supplier's perspective, however, there are again only three relevant contracts, i.e., $C_{H H}^{i}, C_{H L}^{i}$ and $C_{L L}^{i}$.

\section{The game without information sharing}

We first consider the case without any information sharing. The analysis in this case is rather simple as each retailer has only two information sets and the assumptions of standard adverse selection models turn out to be satisfied. More precisely, let denote $\nu_{k m}$ with $k, m \in\{L, H\}$ the conditional probability for $a_{k}$ given signal $s_{m}$, i.e., for example

$$
\nu_{H H}=\frac{\rho_{H} \rho_{H H}}{\rho_{H} \rho_{H H}+\rho_{L} \rho_{H L}} \text { for } a=1 \text {, given signal } s_{H}
$$

and assume that retailer $j$ follows its signal, i.e., chooses $C_{k}^{j}$ if he received $s_{k}$. Then the expected profit of retailer $i$ with signal $s_{H}$ and contract $C_{H}^{i}$ can be written as (the expected

\footnotetext{
${ }^{3}$ In section 5 we show how truthtelling can be induced by an ex-ante balanced payment scheme, which is based on the signal reports.
} 
profit with signal $s_{L}$ is determined analogously)

$$
\begin{aligned}
E \Pi_{i}\left(\cdot, C_{H}^{i}, s_{H}\right) & =\nu_{H H}\left[\rho_{H H}\left(1-x_{H}^{i}-x_{H}^{j}\right) x_{H}^{i}+\rho_{L H}\left(1-x_{H}^{i}-x_{L}^{j}\right) x_{H}^{i}\right] \\
& +\nu_{L H}\left[\rho_{H L}\left(\alpha-x_{H}^{i}-x_{H}^{j}\right) x_{H}^{i}+\rho_{L L}\left(\alpha-x_{H}^{i}-x_{L}^{j}\right) x_{H}^{i}\right]-T_{H}^{i}
\end{aligned}
$$

Analyzing the incentive compatibility and participation conditions, i.e.,

$$
E \Pi_{i}\left(\cdot, C_{H}^{i}, s_{H}\right) \geq E \Pi_{i}\left(\cdot, C_{L}^{i}, s_{H}\right) \text { and } E \Pi_{i}\left(\cdot, C_{L}^{i}, s_{L}\right) \geq E \Pi_{i}\left(\cdot, C_{H}^{i}, s_{L}\right)
$$

shows that the single crossing property holds and that the binding conditions are given by

$$
E \Pi_{i}\left(\cdot, C_{H}^{i}, s_{H}\right)=E \Pi_{i}\left(\cdot, C_{L}^{i}, s_{H}\right) \text { and } E \Pi_{i}\left(\cdot, C_{L}^{i}, s_{L}\right)=0
$$

Employing (1) it is straightforward to determine the optimal contracts for the supplier: Maximizing the supplier's expected profit Eח given by

$$
\begin{aligned}
E \Pi & =\frac{1}{2}\left[\begin{array}{c}
\rho_{H H} \rho_{H H}\left(T_{H}^{1}+T_{H}^{2}\right)+\rho_{H H} \rho_{L H}\left(T_{H}^{1}+T_{L}^{2}\right) \\
+\rho_{H H} \rho_{L H}\left(T_{L}^{1}+T_{H}^{2}\right)+\rho_{L H} \rho_{L H}\left(T_{L}^{1}+T_{L}^{2}\right)
\end{array}\right] \\
& +\frac{1}{2}\left[\begin{array}{c}
\rho_{H L} \rho_{H L}\left(T_{H}^{1}+T_{H}^{2}\right)+\rho_{H L} \rho_{L L}\left(T_{H}^{1}+T_{L}^{2}\right) \\
+\rho_{H L} \rho_{L L}\left(T_{L}^{1}+T_{H}^{2}\right)+\rho_{L L} \rho_{L L}\left(T_{L}^{1}+T_{L}^{2}\right)
\end{array}\right] .
\end{aligned}
$$

we get:

Lemma 1 The optimal (symmetric) quantities without information sharing $X_{H}^{*}(\alpha, \xi)$ and $X_{L}^{*}(\alpha, \xi)$ satisfy $X_{H}^{*}(\alpha, \xi)>X_{L}^{*}(\alpha, \xi)$. While $X_{H}^{*}(\alpha, \xi)$ is undistorted, $X_{L}^{*}(\alpha, \xi)$ is distorted downwards. The optimal payments are such that $T_{H}^{*}(\alpha, \xi) \geq T_{L}^{*}(\alpha, \xi)$ with strict inequality for $\xi \in(0,1]$.

Proof. See Appendix.

The downward distortion of $X_{L}^{*}(\alpha, \xi)$ is due to the observation that the profit from deviating by choosing $C_{L}$ instead of $C_{H}$ is increasing in $x_{L}$. Hence, the incentive compatible payment $T_{H}$ is the higher the lower $x_{L}$ which implies that $X_{L}^{*}(\alpha, \xi)$ is lower than in the case without any binding incentive condition. 
For later reference let $E \Pi^{*}(\cdot)$ denote the retailers' expected profit without information sharing

$$
E \Pi^{*}(\cdot)=\frac{1}{2}\left(\rho_{H H}+\rho_{H L}\right) E \Pi_{i}\left(\cdot, C_{H}^{*}, s_{H}\right)
$$

\section{Information sharing with verifiable Information}

\subsection{Partial Information Sharing $L$}

With partial information $L$ there are three possible signal and information combinations. Thus, the supplier can restrict its offers to three possible contracts $C_{H 0}^{i}, C_{L H}^{i}$ and $C_{L L}^{i}$ (see Figure 1).

Assuming incentive compatibility, retailer $i^{\prime}$ 's expected profit given its own signal is $s_{H}$ and the message is $M^{P L}=(0)$ can be written as

$$
\begin{aligned}
E \Pi_{i}\left(\cdot, C_{H 0}^{i}, s_{H}, M^{P L}=(0)\right)=\nu_{H 0 H}\left[\begin{array}{c}
\rho_{H H}\left(1-x_{H H}^{i}-x_{H H}^{j}\right) x_{H H}^{i}+ \\
\rho_{L H}\left(1-x_{H H}^{i}-x_{H L}^{j}\right) x_{H H}^{i}
\end{array}\right] \\
+\nu_{L 0 H}\left[\begin{array}{l}
\rho_{H L}\left(\alpha-x_{H H}^{i}-x_{H L}^{j}\right) x_{H H}^{i}+ \\
\rho_{L L}\left(\alpha-x_{H H}^{i}-x_{H L}^{j}\right) x_{H L}^{i}
\end{array}\right]-T_{H 0}^{i}
\end{aligned}
$$

where

$$
\nu_{H 0 H}=\frac{\rho_{H} \rho_{H H}}{\rho_{H} \rho_{H H}+\rho_{L} \rho_{H L}} \text { and } \nu_{L 0 H}=1-\nu_{H 0 H}
$$

denote the conditional probability for $a=1$ and $a=\alpha$, respectively, given message $M^{P L}=(0)$ and own signal $s_{H}$. The retailers' expected profits for the other cases can be calculated analogously.

Turning to the retailers' incentive and participation constraints, we proceed by assuming that $x_{H 0}^{i} \geq x_{L H}^{i}, x_{L L}^{i}$ and

$E \Pi_{i}\left(\cdot, C_{H 0}^{i}, s_{H}, M^{P L}=(0)\right) \geq E \Pi_{i}\left(\cdot, C_{L H}^{i}, s_{H}, M^{P L}=(0)\right), E \Pi_{i}\left(\cdot, C_{L L}^{i}, s_{H}, M^{P L}=(0)\right)$ 
is binding. Taking into account the incentive conditions for retailers with signal $s_{L}$, i.e.,

$$
\begin{aligned}
& E \Pi_{i}\left(\cdot, C_{L H}^{i}, s_{L}, M^{P L}=(0)\right) \geq E \Pi_{i}\left(\cdot, C_{L L}^{i}, s_{L}, M^{P L}=(0)\right) \\
& E \Pi_{i}\left(\cdot, C_{L L}^{i}, s_{L}, M^{P L}=\left(s_{L}, s_{L}\right)\right) \geq E \Pi_{i}\left(\cdot, C_{L H}^{i}, s_{L}, M^{P L}=\left(s_{L}, s_{L}\right)\right)
\end{aligned}
$$

and solving the maximization problem of the supplier, we get that the optimal contracts of the supplier duplicate the contracts offered without information sharing:

Lemma 2 The optimal (symmetric) quantities with partial information sharing $L$, denoted by $X_{H 0}^{P L}(\alpha, \xi), X_{L H}^{P L}(\alpha, \xi)$ and $X_{L L}^{P L}(\alpha, \xi)$, are such that

$$
X_{H 0}^{P L}(\alpha, \xi)=X_{H}^{*}(\alpha, \xi) \text { and } X_{L H}^{P L}(\alpha, \xi)=X_{L L}^{P L}(\alpha, \xi)=X_{L}^{*}(\alpha, \xi)
$$

The induced fixed payments and the retailers' expected profits are the same as in the case without information sharing.

Intuitively, partial information sharing $L$ does not change the information of retailers with signal $s_{H}$. With $s^{i}=s_{L}$ and $M^{P L}=(0)$ retailer $i$ knows that retailer $j$ will offer the high quantity $X_{H 0}$. However, the retailer with $s_{L}$ has a higher posterior for high demand due to the other retailer receiving signal $s_{H}$. With $M^{P L}=\left(s_{L}, s_{L}\right)$ both retailers know that they both got bad signals which implies that the posterior probability for high demand is low. However, they know that both of them will choose to supply a low quantity. As in any case the countervailing effects on expected demand and supply by the other retailer cancel, the optimal response of the supplier is to design his contracts such that this information is ignored. Therefore, he offers the same contracts as in the case of no information sharing.

\subsection{Partial Information Sharing $H$}

In contrast to above, information sharing $H$ induces the supplier to offer different contracts than in case of no information sharing. Intuitively, information sharing $H$ is "good news" as in expectation the possible information rent is higher due to the case of one retailer having signal $s_{H}$, while the other retailer having signal $s_{L}$. In this case the retailer with signal $s_{H}$ has information for high demand, and can infer the other retailer's low demand signal. In contrast, the retailer with signal $s_{L}$ cannot make any inference, leading to an 
informational advantage for the retailer with signal $s_{H}$. Exploiting this advantage leads to a relatively high information rents in this scenario.

There are again three possible signal and information combinations. Thus, the supplier can restrict its offers to three contracts $C_{H H}^{i}, C_{H L}^{i}$ and $C_{L 0}^{i}$ (see Figure 1).

Assuming incentive compatibility, the retailer $i^{\prime}$ 's expected profit given its own signal is $s_{H}$ and the message is $M^{H}=\left(s_{H}, s_{H}\right)$ can be written as

$$
\begin{aligned}
E \Pi_{i}\left(\cdot, C_{H H}^{i}, s_{H}, M^{H}=\left(s_{H}, s_{H}\right)\right)= & \nu_{H H H}\left(1-x_{H H}^{i}-x_{H H}^{j}\right) x_{H H}^{i} \\
& +\nu_{L H H}\left(\alpha-x_{H H}^{i}-x_{H H}^{j}\right) x_{H H}^{i}-T_{H H}^{i}
\end{aligned}
$$

where

$$
\nu_{H H H}=\frac{\rho_{H} \rho_{H H} \rho_{H H}}{\rho_{H} \rho_{H H} \rho_{H H}+\rho_{L} \rho_{H L} \rho_{H L}} \text { and } \nu_{L H H}=1-\nu_{H H H}
$$

denote the conditional probability for $a=1$ and $a=\alpha$, respectively, given $M^{H}=\left(s_{H}, s_{H}\right)$. The retailers' expected profits for the other cases can be calculated analogously.

Turning to the retailers' incentive and participation constraints, we proceed by assuming that $x_{H L}^{i} \geq x_{H H}^{i}$ holds and that the incentive conditions are binding downwards, i.e., that the optimal contracts offered by the supplier satisfy

$$
\begin{aligned}
& E \Pi_{i}\left(\cdot, C_{H L}^{i}, s_{H}, M^{P H}=(0)\right)=E \Pi_{i}\left(\cdot, C_{H H}^{i}, s_{H}, M^{P H}=(0)\right) \\
& E \Pi_{i}\left(\cdot, C_{H H}^{i}, s_{H}, M^{P H}=\left(s_{H}, s_{H}\right)\right)=E \Pi_{i}\left(\cdot, C_{L 0}^{i}, s_{H}, M^{P H}=\left(s_{H}, s_{H}\right)\right) \\
& E \Pi_{i}\left(\cdot, C_{L 0}^{i}, s_{L}, M^{P H}=(0)\right)=0
\end{aligned}
$$

Using (8) - (10) maximization of the supplier's expected profits leads to

Lemma 3 The optimal (symmetric) quantities with partial information sharing $H$, denoted by $X_{H L}^{P H}(\alpha, \xi), X_{H H}^{P H}(\alpha, \xi)$ and $X_{L 0}^{P H}(\alpha, \xi)$, are such that $X_{H L}^{P H}(\alpha, \xi)>X_{H H}^{P H}(\alpha, \xi)>$ $X_{L 0}^{P H}(\alpha, \xi) \cdot X_{H L}^{P H}(\alpha, \xi)$ is not distorted, $X_{H H}^{P H}(\alpha, \xi)$ and $X_{L 0}^{P H}(\alpha, \xi)$ are distorted downwards. The optimal payments satisfy $T_{H L}^{P H}(\alpha, \xi) \geq T_{H H}^{P H}(\alpha, \xi) \geq T_{L 0}^{P H}(\alpha, \xi)$ with strict inequalities for $\xi>0$.

The distortion results are based on the same reasoning as in the case of no information sharing. Analyzing the payments $T_{H H}, T_{L 0}$ and $T_{H L}$ implied by (8)-(10) shows that $x_{L H}$ 
does not affect $T_{H H}$ and $T_{L 0}$, whereas $T_{H L}$ is decreasing in $x_{H H}$ and $x_{L 0}$. Additionally, $x_{L 0}$ also affects $T_{H H}$ negatively.

Let $E \Pi^{P H}(\alpha, \xi)$ denote the expected profit of the retailers with partial information sharing $H$ :

$$
\begin{aligned}
E \Pi^{P H}(\cdot)= & \frac{1}{2}\left(\rho_{H H} \rho_{H H}+\rho_{H L} \rho_{H L}\right) E \Pi_{i}\left(\cdot, C_{H H}^{P H}, s_{H}, M^{P H}=\left(s_{H}, s_{H}\right)\right) \\
& +\frac{1}{2}\left(\rho_{H H} \rho_{L H}+\rho_{H L} \rho_{L L}\right) E \Pi_{i}\left(\cdot, C_{H L}^{P H}, s_{H}, M^{P H}=(0)\right)
\end{aligned}
$$

\subsection{Complete Information Sharing}

Assume that retailer $j$ follows its signals, i.e., chooses $C_{k m}^{j}$ if he receives the message $\left(s_{k}, s_{m}\right)$. The expected profit of retailer $i$ if the received signals are $s_{H}$ and $s_{H}$ and the chosen contract is $C_{H H}^{i}$ is again given by (see (7))

$$
\begin{aligned}
E \Pi_{i}\left(\cdot, C_{H H}^{i}, M^{C}=\left(s_{H}, s_{H}\right)\right)= & \nu_{H H H}\left(1-x_{H H}^{i}-x_{H H}^{j}\right) x_{H H}^{i} \\
& +\nu_{L H H}\left(\alpha-x_{H H}^{i}-x_{H H}^{j}\right) x_{H H}^{i}-T_{H H}^{j}
\end{aligned}
$$

In order to proceed, note first that the single crossing property does not hold for $E \Pi_{i}\left(\cdot, C_{H H}^{1}, s_{H}, s_{H}\right)$ and $E \Pi_{i}\left(\cdot, C_{H L}^{i}, s_{H}, s_{H}\right)$ as well as for $E \Pi_{i}\left(\cdot, C_{H L}^{i}, s_{H}, s_{L}\right)$ and $E \Pi_{i}\left(\cdot, C_{L L}^{i}, s_{H}, s_{L}\right)$. We assume that the incentive conditions are downward binding, i.e.,

$$
\begin{aligned}
& E \Pi_{i}\left(\cdot, C_{H H}^{i}, M^{C}=\left(s_{H}, s_{H}\right)\right)=E \Pi_{i}\left(\cdot, C_{H L}^{i}, M^{C}=\left(s_{H}, s_{H}\right)\right) \\
& E \Pi_{i}\left(\cdot, C_{H L}^{i}, M^{C}=\left(s_{H}, s_{L}\right)\right)=E \Pi_{i}\left(\cdot, C_{L L}^{i}, M^{C}=\left(s_{H}, s_{L}\right)\right) \text { and } \\
& E \Pi_{i}\left(\cdot, C_{L L}^{i}, M^{C}=\left(s_{L}, s_{L}\right)\right)=0
\end{aligned}
$$

and check whether the solutions lead to

$$
\begin{aligned}
& E \Pi_{i}\left(\cdot, C_{H L}^{C}, M^{C}=\left(s_{H}, s_{L}\right)\right) \geq E \Pi_{i}\left(\cdot, C_{H H}^{C}, M^{C}=\left(s_{H}, s_{L}\right)\right) \text { and } \\
& E \Pi_{i}\left(\cdot, C_{L L}^{i}, M^{C}=\left(s_{L}, s_{L}\right)\right) \geq E \Pi_{i}\left(\cdot, C_{H L}^{i}, M^{C}=\left(s_{L}, s_{L}\right)\right), E \Pi_{i}\left(\cdot, C_{H H}^{i}, M^{C}=\left(s_{L}, s_{L}\right)\right)
\end{aligned}
$$

Using (13)—(15) and solving for the optimal quantities we get

Lemma 4 With complete information sharing, the optimal (symmetric) quantities 
$X_{H H}^{C}(\alpha, \xi), X_{H L}^{C}(\alpha, \xi)$ and $X_{L L}^{C}(\alpha, \xi)$ are such that $X_{H H}^{C}(\alpha, \xi)>X_{H L}^{C}(\alpha, \xi)>X_{L L}^{C}(\alpha, \xi)$. While $X_{H H}^{C}(\alpha, \xi)$ is undistorted, $X_{H L}^{C}(\alpha, \xi)$ and $X_{L L}^{C}(\alpha, \xi)$ are distorted downwards. The optimal payments satisfy $T_{H H}^{C}(\alpha, \xi) \geq T_{H L}^{C}(\alpha, \xi) \geq T_{L L}^{C}(\alpha, \xi)$ with strict inequalities for $\xi \in(0,1]$.

Again, the payments $T_{H H}, T_{H L}$ and $T_{L L}$ which solve (13)-(15) are such that $x_{H H}$ does not affect $T_{H L}$ and $T_{L L}$, whereas $T_{H H}$ is decreasing in $x_{H L}$ and $x_{L L}$. Additionally, $T_{H L}$ decreases in $x_{L L}$.

Let $E \Pi^{c}(\alpha, \xi)$ denote the expected profit of the retailers with complete information sharing:

$$
\begin{aligned}
E \Pi^{C}(\cdot) & =\frac{1}{2}\left(\rho_{H H} \rho_{H H}+\rho_{H L} \rho_{H L}\right) E \Pi_{i}\left(\cdot, C_{H H}^{C}, M^{C}=\left(s_{H}, s_{H}\right)\right) \\
& +\left(\rho_{H H} \rho_{L H}+\rho_{H L} \rho_{L L}\right) E \Pi_{i}\left(\cdot, C_{H L}^{C}, M^{C}=\left(s_{L}, s_{H}\right)\right)
\end{aligned}
$$

\subsection{Comparison of the optimal quantities}

Before we proceed with analyzing the retailers' incentives to report their signals truthfully in the next section, we compare the optimal quantities under the different information sharing agreements. As already mentioned, information sharing $L$ leads to the same allocations as no information sharing.

Lemma 5 The optimal quantities under the different information structures satisfy

$$
\begin{aligned}
& X_{H L}^{P H}(\alpha, \xi)>X_{H H}^{C}(\alpha, \xi)=X_{H}^{*}(\alpha, \xi)>X_{H H}^{P H}(\alpha, \xi), \\
& X_{H H}^{P H}(\alpha, \xi) \gtreqless X_{H L}^{C}(\alpha, \xi) \Leftrightarrow 175 \xi^{2}-95 \xi^{4}+17 \xi^{6} \gtreqless 1 \\
& X_{L}^{*}(\alpha, \xi) \gtreqless X_{L L}^{C}(\alpha, \xi) \Leftrightarrow \xi \lesseqgtr 1 / \sqrt{3} \text { and } \\
& X_{L}^{*}(\alpha, \xi), X_{L L}^{C}(\alpha, \xi)>X_{L 0}^{P H}(\alpha, \xi)
\end{aligned}
$$

Given a retailer receives signal $s_{H}$, his willingness to pay for a high quantity is higher, if he knows that the other retailer has signal $s_{L}$, as if he does not know the other retailer's signal or if this signal is $s_{H}$ as well. This leads to $X_{H L}^{P H}(\alpha, \xi)>X_{H H}^{C}(\alpha, \xi)$. The best response to the high quantity $X_{H L}^{P H}$, is a low quantity $X_{L 0}^{P H}$ which is further decreased to soften the incentive constraint for the case in which both retailers receive $s_{H}$ (see (9)). 
Considering the total quantities offered to consumers, we get:

Corollary 1 Compared to the case with no information sharing, partial information sharing unambiguously reduces the quantities offered to consumers,.i.e.,

$$
X_{H}^{*}(\alpha, \xi)+X_{L}^{*}(\alpha, \xi)>X_{H L}^{P H}(\alpha, \xi)+X_{L 0}^{P H}(\alpha, \xi)
$$

Complete information sharing does not affect the quantities offered; if both retailers receive $s_{H}$. In the other cases the total quantities are either lower or higher, i.e.,

$$
\begin{aligned}
& X_{H}^{*}(\alpha, \xi)+X_{L}^{*}(\alpha, \xi) \gtreqless 2 X_{H L}^{C}(\alpha, \xi) \Leftrightarrow \xi \gtreqless 1 / \sqrt{3} \\
& X_{L}^{*}(\alpha, \xi) \gtreqless X_{L L}^{C}(\alpha, \xi) \Leftrightarrow \xi \lesseqgtr 1 / \sqrt{3}
\end{aligned}
$$

For consumers partial information sharing is never beneficial. In case of low (both retailers receiving $s_{L}$ ) or high (both retailers receiving $s_{H}$ ) demand expectations, lemma 5 already indicates that total quantities are higher without information sharing. But also in case of intermediate demand expectations (one retailer receiving $s_{H}$ and one retailer receiving $\left.s_{L}\right)$ the total quantity is lower than without information sharing. This last result is due to the downward distortions of $X_{L 0}^{P H}$.

However, while the effects of complete information sharing are ambiguous for intermediate and low demand expectations, quantities do not change due to information sharing, if demand expectations are high.

\section{$5 \quad$ Inducing truth-telling}

Turning to the incentives of the retailers to truthfully reveal their signal, one might argue that each retailer has an incentive to deviate by always reporting $s_{L}$ in order to induce the other retailer to believe $a=\alpha$ and thus to choose a contract with a lower quantity. This argument is certainly true for complete information sharing where reporting $s_{L}$ instead of $s_{H}$ will induce the other retailer to choose either $C_{H L}^{C}$ or $C_{L L}^{C}$. With partial information sharing $H$, however, reporting $s_{H}$ instead of $s_{L}$ either does not change the decision of the other retailer or induces the other retailer to choose $C_{H H}^{P H}$ instead of $C_{H L}^{P H}$. 
This together with $X_{H L}^{P H} \geq X_{H H}^{P H}$ decreases the quantity the other retailer offers. In fact, it turns out that the binding constraint for the payment scheme is that each retailer must not have an incentive to report $s_{H}$ instead of $s_{L}$.

In the following we will characterize simple payment schemes which induce truth telling and which are ex ante balanced, i.e., which do not lead to burning any money in expectation.

\subsection{Partial Information Sharing $H$}

Note first that with partial information sharing $H$ the payment of each retailer has to be based on the retailer's signal only. This is simply due to the fact that payments based on both signals would lead to full information sharing.

Ex ante balance of the payment scheme requires

$$
E z=\frac{1}{2}\left(\rho_{H H}+\rho_{H L}\right) z_{H}+\frac{1}{2}\left(\rho_{L H}+\rho_{L L}\right) z_{L}=0 \Rightarrow z_{L}=-z_{H}
$$

where $z_{k}$ denotes the payment a retailer has to make if he reports $s_{k}$.

Since the decisions whether the retailers report their signal truthfully takes place after receiving their signals we need to calculate the retailers' expected profits in stage four of the game, i.e., the stage in which retailers - given their signals - decide which signal they report to the third party.

For example, assume that retailer $i$ receives signal $s_{H}$. Then, his expected profit with truth telling, i.e., $\forall i: \widetilde{s}_{i}=s_{i}$, can be written as

$$
\begin{aligned}
E \Pi_{i}^{P H}\left(\cdot, s_{H}, \widetilde{s}^{i}\right. & \left.=s_{H}\right)=\mu_{H H H}\left[\left(1-X_{H H}^{P H}-X_{H H}^{P H}\right) X_{H H}^{P H}-T_{H H}^{P H}\right] \\
& +\mu_{H L H}\left[\left(1-X_{H L}^{P H}-X_{L 0}^{P H}\right) X_{H L}^{P}-T_{H L}^{P H}\right] \\
& +\mu_{L H H}\left[\left(\alpha-X_{H H}^{P H}-X_{H H}^{P H}\right) X_{H H}^{P}-T_{H H}^{P H}\right] \\
& +\mu_{L L H}\left[\left(\alpha-X_{H L}^{P H}-X_{L 0}^{P H}\right) X_{H L}^{P H}-T_{H L}^{P H}\right]-z_{H}
\end{aligned}
$$

where $\mu_{m k l}$ with $m, k, l \in\{H, L\}$ denotes the conditional probability of $a=a_{m}$ and $s^{j}=s_{k}$ given $s^{i}=s_{l}$. Deviating by reporting $s_{L}$ instead of $s_{H}$ and choosing contract $C_{H H}^{P H}$ would lead to (note that reporting $s_{L}$ implies that the retailer remains uninformed about the 
signal of the other retailer since $M^{P H}=(0)$ in any case)

$$
\begin{aligned}
E \Pi_{i}\left(C_{H H}^{P H}, s_{H}, \widetilde{s}^{i}\right. & \left.=s_{L}\right)=\mu_{H H H}\left[\left(1-X_{H H}^{P H}-X_{H L}^{P H}\right) X_{H H}^{P H}-T_{H H}^{P H}\right] \\
& +\mu_{H L H}\left[\left(1-X_{H H}^{P H}-X_{L 0}^{P H}\right) X_{H L}^{P H}-T_{H H}^{P H}\right] \\
& +\mu_{L H H}\left[\left(\alpha-X_{H H}^{P H}-X_{H L}^{P H}\right) X_{H H}^{P H}-T_{H H}^{P H}\right] \\
& +\mu_{L L H}\left[\left(\alpha-X_{H H}^{P H}-X_{L 0}^{P H}\right) X_{H L}^{P H}-T_{H H}^{P H}\right]-z_{L}
\end{aligned}
$$

The other possible deviation strategies - given signal $s_{H}$ - are to report $s_{L}$ and to choose either $\left(T_{H L}^{P H}, x_{H L}^{P H}\right)$ or $\left(T_{L 0}^{P H}, x_{L 0}^{P H}\right)$. Similarly, with signal $s_{L}$ a retailer can deviate by reporting $s_{H}$ and by choosing either $\left(T_{H H}^{P H}, x_{H H}^{P H}\right),\left(T_{H L}^{P H}, x_{H L}^{P H}\right)$ or $\left(T_{L 0}^{P H}, x_{L 0}^{P H}\right)$ depending on wether $M^{P H}=\left(s_{H}, s_{H}\right)$ or if $M^{P H}=(0)$.

Comparing the expected profits for all possible deviation strategies it turns out that the binding constraint is such that truthfully reporting $s_{L}$ and choosing $C_{L 0}^{P H}$ must not be dominated by reporting $s_{H}$ and choosing $C_{L 0}^{P H}$ irrespectively of the message sent back from the third party, i.e., we must have

$$
\begin{aligned}
E \Pi_{i}\left(\cdot, s_{L}, \widetilde{s}^{i}=s_{L}\right)= & E \Pi_{i}\left(C_{L 0}^{P H}, s_{L}, \widetilde{s}^{i}=s_{H}\right) \\
= & \mu_{H H L}\left[\left(1-X_{L 0}^{P H}-X_{H H}^{P H}\right) X_{L 0}^{P H}-T_{L O}^{P H}\right] \\
& +\mu_{H L L}\left[\left(1-X_{L 0}^{P H}-X_{L 0}^{P H}\right) X_{L 0}^{P H}-T_{L O}^{P H}\right] \\
& +\mu_{L H L}\left[\left(\alpha-X_{L 0}^{P H}-X_{H H}^{P H}\right) X_{L 0}^{P H}-T_{L O}^{P H}\right] \\
& +\mu_{L L L}\left[\left(\alpha-X_{L 0}^{P H}-X_{L 0}^{P H}\right) X_{L 0}^{P H}-T_{L O}^{P H}\right]-z_{H}
\end{aligned}
$$

Solving for the implied payment leads to

$$
z_{H}^{P H}=(1-\alpha) \xi\left(3-\xi^{2}\right)\left(1-\xi^{2}\right)^{2} \frac{3(1+\alpha)-32(1-\alpha) \xi+12(1+\alpha) \xi^{2}+(1+\alpha) \xi^{4}}{24\left(1+\xi^{2}\right)\left(3+12 \xi^{2}+\xi^{4}\right)^{2}}>0
$$




\subsection{Complete Information Sharing}

Turning to complete information sharing, let $z_{k m}$ denote the payment of retailer $i$ if he reports $s_{m}$ and the other retailer reports $s_{k}$. Ex ante balance of the payments requires

$$
\begin{aligned}
E z= & \frac{1}{2}\left(\rho_{H H} \rho_{H H} z_{H H}+\rho_{L H} \rho_{H H}\left(z_{H L}+z_{L H}\right)+\rho_{L H} \rho_{L H} z_{L L}\right) \\
& +\frac{1}{2}\left(\rho_{H L} \rho_{H L} z_{H H}+\rho_{H L} \rho_{L L}\left(z_{H L}+z_{L H}\right)+\rho_{L L} \rho_{L L} z_{L L}\right)=0
\end{aligned}
$$

When deciding which signals to report, we again have to consider all possible deviation strategies. Comparing the respective deviation profits we get that the optimal deviation strategy if retailer $i^{\prime}$ s own signal is $s_{H}$ is to report $\widetilde{s}^{i}=s_{L}$ and to choose

$C_{H H}$ if the retailer $j$ reports $s_{H}$ and $\begin{cases}C_{H H} & \text { if retailer } j \text { reports } s_{L} \text { and } \xi \leq \frac{1}{\sqrt{3}} \\ C_{H L} & \text { if retailer } j \text { reports } s_{L} \text { and } \xi \geq \frac{1}{\sqrt{3}}\end{cases}$

Let $E \Pi_{i}^{D *}\left(\cdot, s_{H}\right)$ denote the optimal deviation profit given signal $s_{H}$.

Similarly, suppose that retailer $i$ has gotten signal $s_{L}$ but reports $s_{H}$. Then, the optimal deviation strategy is to choose $C_{L L}$ irrespective of the report retailer $j$ sends. Let $E \Pi_{i}^{D *}\left(\cdot, s_{L}\right)$ denote the optimal deviation profit given signal $s_{L}$.

In order to guarantee truth-telling and thus (honest) information sharing between the retailers, the payments have to be such that the following two inequalities are satisfied

$$
E \Pi_{i}^{C}\left(\cdot, s_{H}\right) \geq E \Pi_{i}^{D *}\left(\cdot, s_{H}\right) \text { and } E \Pi_{i}^{C}\left(\cdot, s_{L}\right) \geq E \Pi_{i}^{D *}\left(\cdot, s_{L}\right)
$$

Assume that $E \Pi_{i}^{C}\left(\cdot, s_{H}\right)=E \Pi_{i}^{D *}\left(\cdot, s_{H}\right)$ and $E \Pi_{i}^{C}\left(\cdot, s_{L}\right)=E \Pi_{i}^{D *}\left(\cdot, s_{L}\right)$ and let $z_{L H}^{C}$ and $z_{H L}^{C}$ denote the solutions of (19) as functions of $z_{L L}$ and $z_{H H}$ and all other parameters. Using (18) to solve for $z_{H H}$ we are left with $z_{L L}$ which we can normalize to 0 since the payment scheme is balanced. The exact values of the payments $z_{H H}^{C}, z_{H L}^{C}$ and $z_{L H}^{C}$ are given in the appendix. 


\section{Optimal Information Sharing and Social Welfare}

Our final step is to analyze whether it is optimal for the retailers to implement partial or complete information sharing. For this purpose we simply have to compare the retailers' profit under either sharing agreements.

Proposition 1 The retailers agree on partial information as long as $\xi$ is sufficiently low. Complete information sharing is optimal for $\xi$ sufficiently high. No information sharing is optimal for intermediate values of $\xi$.

The different regions mentioned in the proposition are shown in Figure 2.

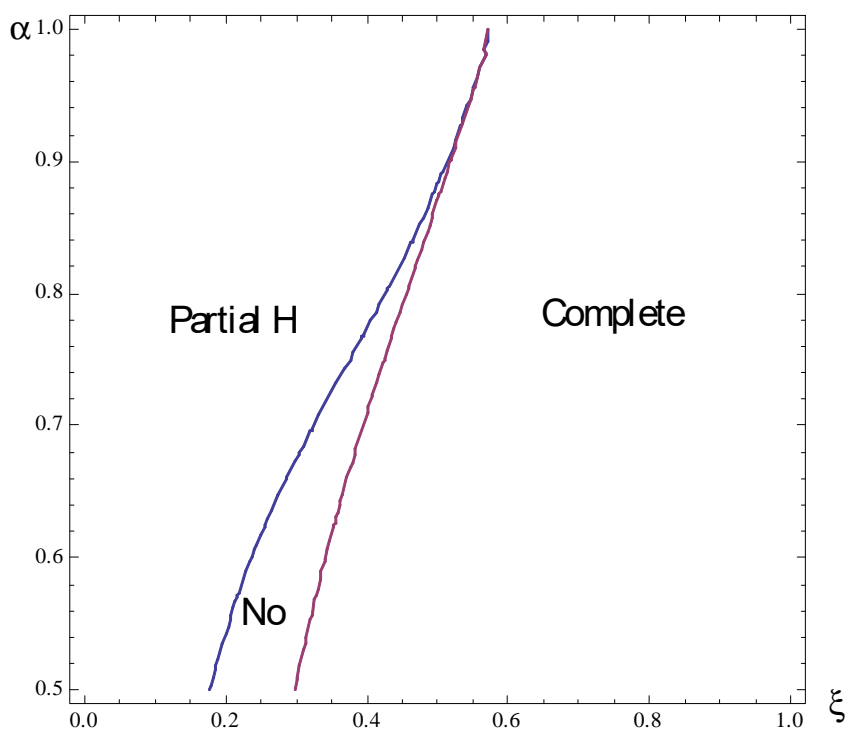

Figure 2: Optimal Information Sharing

Consider first the comparison between partial information sharing $H$ and no information sharing. Partial information sharing $H$ is beneficial for a retailer in case he receives a signal $s_{H}$ and a message $M^{P H}=(0)$ as he gets fully informed about both signals. Accordingly, in this case his profits, i.e., his information rent, is higher as his rent with signal $s_{H}$ and no information sharing. On the other hand, receiving signal $s_{H}$ and message $M^{P H}=$ $\left(s_{H}, s_{H}\right)$ leads to lower profits then receiving signal $s_{H}$ without informations sharing. As the probability for this later case increases with $\xi$, no information sharing is better than partial information if $\xi$ is sufficiently small.

A similar argument holds for the comparison between partial information sharing $H$ and complete information sharing. Again, while partial information sharing on $H$ is beneficial 
for a retailer, if he receives a signal $s_{H}$ and a message $M^{P H}=(0)$, he would prefer complete information sharing if he receives a signal $s_{L}$. With signal $s_{L}$ he gets a zero profit under partial information sharing $H$ whereas with complete information sharing his expected profits are strictly positive. If $\xi$ is sufficiently small, the first of the effects dominates the second, making partial information sharing more desirable.

Considering social welfare we get that there is only a small region where the behavior of the retailers is in line with welfare considerations. More precisely, let $E W^{*}, E W^{P H}$ and $E W^{C}$ denote expected social welfare measured as the sum of expected consumer surplus and supplier's and retailers' expected profits with no information sharing $(*)$, partial information sharing $H(P H)$ and complete information sharing $(C)$, respectively. Then, we get

Proposition 2 Complete information sharing is socially optimal for $\xi<1 / \sqrt{3}$; for $\xi>$ $1 / \sqrt{3}$ no information sharing is socially desirable:

$$
E W^{*}>E W^{P H} \text { for all } \xi \text { and } E W^{*} \gtreqless E W^{C} \Leftrightarrow \xi \gtreqless 1 / \sqrt{3}
$$

Proposition 2 resembles Corollary 1 and shows that the distortions induced by partial information sharing always lead to lower welfare as compared to no information sharing. Comparing no information sharing and full information sharing, the welfare gain due to $X_{H}^{*}(\alpha, \xi)+X_{L}^{*}(\alpha, \xi)>2 X_{H L}^{C}(\alpha, \xi)$ more then compensates the welfare loss due to $2 X_{L}^{*}(\alpha, \xi)<2 X_{L L}^{C}(\alpha, \xi)$ if the signal precision is sufficiently high, i.e., if $\xi>1 / \sqrt{3}$. However, if $\xi<1 / \sqrt{3}$ it holds that $X_{H}^{*}(\alpha, \xi)+X_{L}^{*}(\alpha, \xi)<2 X_{H L}^{C}(\alpha, \xi)$ and then both effects are in favor of information sharing.

Figure 3 combines Figure 2 and Proposition 2. The shaded area shows the region in which the retailers' decision to fully share their information is in line with social welfare considerations. Outside this region, the retailers either share too much information $(\xi$ sufficiently high) or too less information ( $\xi$ sufficiently low). 


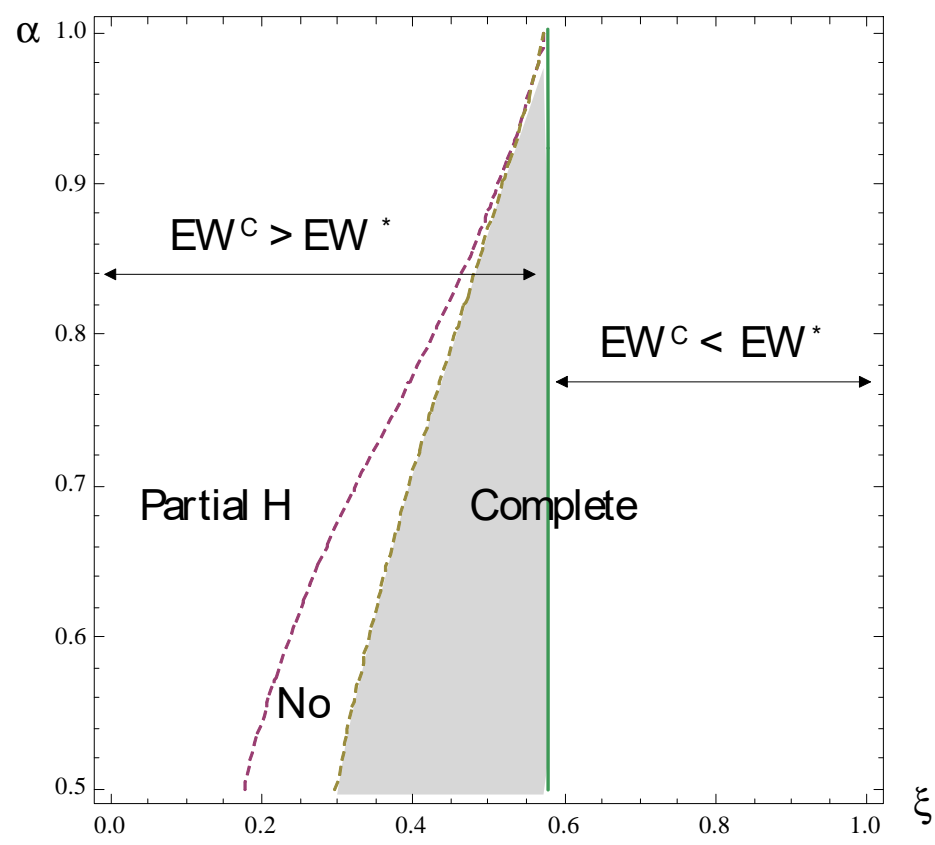

Figure 3: Expected social welfare

\section{Conclusion}

In this model we have shown that in a two-tier industry firms do have incentives to fully share information as long as their private signals are sufficiently informative. However, only for an intermediate range of this signal precision the firms' sharing agreements are also socially desirable. Thus, looking at the literature on countervailing power, one should note that it is not only collective bargaining, which allows the retailers to exert pressure on the supplier, but also some more intricate devices as different regimes of information sharing, which allow them to increase their rents. Therefore policy evaluation in this domain should not look only at the immediate effects of coordination, but consider more complex variants of cooperation among firms.

\section{Appendix}

\subsection{Proofs}

Proof of Lemma $1 \quad$ Using the solutions for $T_{H}^{i}$ and $T_{L}^{i}$ from solving (1) the optimal quantities follow from maximizing (2) with respect to $x_{H}^{i}$ and $x_{L}^{i}$ and solving the respective 
first order conditions using symmetry. The optimal symmetric quantities are given by

$$
\begin{aligned}
& X_{H}^{*}(\alpha, \xi)=\frac{1}{6}\left(1+\alpha-\frac{2(1-\alpha) \xi}{1+\xi^{2}}\right) \\
& X_{L}^{*}(\alpha, \xi)=\frac{1}{6}\left(1+\alpha-\frac{4(1-\alpha) \xi}{1+\xi^{2}}\right) .
\end{aligned}
$$

The no distortion result for $X_{H}^{*}(\alpha, \xi)$ follows from $\partial T_{L}^{i} / \partial x_{H}^{i}=0$. On the other hand, evaluating $\partial T_{H}^{i} / \partial x_{L}^{i}$ at the equilibrium quantities leads to $\partial T_{H}^{i} / \partial x_{L}^{i}<0$ and thus to a downward distortion.

Proof of Lemma 2 In order to prove the lemma, assume first that the binding conditions are given by

$$
\begin{aligned}
& E \Pi_{i}\left(\cdot, C_{H 0}^{i}, s_{H}, M^{P L}=(0)\right)=E \Pi_{i}\left(\cdot, C_{L H}^{i}, s_{H}, M^{P L}=(0)\right) \\
& E \Pi_{i}\left(\cdot, C_{L H}^{i}, s_{L}, M^{P L}=(0)\right)=E \Pi_{i}\left(\cdot, C_{L L}^{i}, s_{L}, M^{P L}=(0)\right) \text { and } \\
& E \Pi_{i}\left(\cdot, C_{L L}^{i}, s_{L}, M^{P L}=\left(s_{L}, s_{L}\right)\right)=0
\end{aligned}
$$

Then, solving the supplier's optimization problem leads to

$$
E \Pi_{i}\left(\cdot, C_{L H}^{i}, s_{L}, M^{P L}=\left(s_{L}, s_{L}\right)\right)>E \Pi_{i}\left(\cdot, C_{L L}^{i}, s_{L}, M^{P L}=\left(s_{L}, s_{L}\right)\right)
$$

Similarly, assume that the binding conditions are given by

$$
\begin{aligned}
& E \Pi_{i}\left(\cdot, C_{H 0}^{i}, s_{H}, M^{P L}=(0)\right)=E \Pi_{i}\left(\cdot, C_{L L}^{i}, s_{H}, M^{P L}=(0)\right) \\
& E \Pi_{i}\left(\cdot, C_{L L}^{i}, s_{L}, M^{P L}=\left(s_{L}, s_{L}\right)\right)=E \Pi_{i}\left(\cdot, C_{L H}^{i}, s_{L}, M^{P L}=\left(s_{L}, s_{L}\right)\right) \text { and } \\
& E \Pi_{i}\left(\cdot, C_{L H}^{i}, s_{L}, M^{P L}=(0)\right)=0
\end{aligned}
$$

Then, solving the supplier's optimization problem leads to

$$
E \Pi_{i}\left(\cdot, C_{L L}^{i}, s_{L}, M^{P L}=(0)\right)>E \Pi_{i}\left(\cdot, C_{L H}^{i}, s_{L}, M^{P L}=(0)\right)
$$

which again contradicts incentive compatibility. Therefore, the optimal contracts of the supplier must be such that there is no information sharing induced separation between 
retailers with signals $s_{L}$, i.e., the messages $M^{P L}=(0)$ and $M^{P L}=\left(s_{L}, s_{L}\right)$ do not affect the offered contracts and we have $X_{L L}^{P L}(\alpha, \xi)=X_{L H}^{P L}(\alpha, \xi)$. Since this also implies that the supplier offers only two contracts, the optimal contracts must duplicate the optimal contracts in the case of no information sharing.

Proof of Lemma 3 The expected profit of the supplier in the case with partial information $H$ sharing is given by

$$
\begin{aligned}
E \Pi(\cdot) & =\frac{1}{2}\left[\rho_{H H} \rho_{H H}\left(T_{H H}^{1}+T_{H H}^{2}\right)+2 \rho_{H H} \rho_{L H}\left(T_{H L}^{1}+T_{L 0}^{2}\right)+\rho_{L H} \rho_{L H}\left(T_{L 0}^{1}+T_{L 0}^{2}\right)\right] \\
& +\frac{1}{2}\left[\rho_{H L} \rho_{H L}\left(T_{H H}^{1}+T_{H H}^{2}\right)+2 \rho_{H L} \rho_{L L}\left(T_{H L}^{1}+T_{L 0}^{2}\right)+\rho_{L L} \rho_{L L}\left(T_{L 0}^{1}+T_{L 0}^{2}\right)\right]
\end{aligned}
$$

Using the solutions for $T_{H L}^{1}, T_{H H}^{1}$ and $T_{L 0}^{1}$ from solving (8)-(10) the optimal quantities follow from maximizing (22):

$$
\begin{aligned}
& X_{H L}^{P H}(\alpha, \xi)=\frac{1}{6}\left(1+\alpha+\frac{16(1-\alpha) \xi}{3+12 \xi^{2}+\xi^{4}}\right) \\
& X_{H H}^{P H}(\alpha, \xi)=\frac{1}{6}\left(1+\alpha+\frac{6(1-\alpha) \xi}{1+\xi^{2}}-\frac{4(1-\alpha) \xi\left(5+3 \xi^{2}\right)}{3+12 \xi^{2}+\xi^{4}}\right) \\
& X_{L 0}^{P H}(\alpha, \xi)=\frac{1}{6}\left(1+\alpha-\frac{32(1-\alpha) \xi}{3+12 \xi^{2}+\xi^{4}}\right)
\end{aligned}
$$

Employing (23) - (25) it is straight forward that the remaining incentive conditions, i.e.,

$$
\begin{aligned}
& E \Pi_{1}\left(\cdot, C_{H H}^{1}, s_{H}, M^{P H}=\left(s_{H}, s_{H}\right)\right) \geq E \Pi_{1}\left(\cdot, C_{H L}^{1}, s_{H}, M^{P H}=\left(s_{H}, s_{H}\right)\right) \\
& E \Pi_{1}\left(\cdot, C_{H L}^{1}, s_{H}, M^{P H}=(0)\right) \geq E \Pi_{1}\left(\cdot, C_{L 0}^{1}, s_{H}, M^{P H}=(0)\right) \\
& E \Pi_{1}\left(\cdot, C_{L 0}^{1}, s_{L}, M^{P H}=(0)\right) \geq E \Pi_{1}\left(\cdot, C_{H H}^{1}, s_{L}, M^{P H}=(0)\right), E \Pi_{1}\left(\cdot, C_{H L}^{1}, s_{L}, M^{P H}=(0)\right),
\end{aligned}
$$

are satisfied. Furthermore, there is no other combination of binding constraints which can be optimal.

Turning to the distortion results, we have $\partial T_{H H}^{i} / \partial x_{H L}^{i}=\partial T_{L 0}^{i} / \partial x_{H L}^{i}=0$. Evaluating $\partial T_{H L}^{i} / \partial x_{H H}^{i}, \partial T_{H L}^{i} / \partial x_{L 0}^{i}$ and $\partial T_{H H}^{i} / \partial x_{L 0}^{i}$ at the equilibrium quantities we get

$$
\frac{\partial T_{H L}^{i}}{\partial x_{H H}^{i}}<0, \frac{\partial T_{H L}^{i}}{\partial x_{L 0}^{i}}<0 \text { as well as } \frac{\partial T_{H H}^{i}}{\partial x_{L 0}^{i}}<0
$$


Proof of Lemma 4 With complete information sharing the expected profit of the supplier can be written as

$$
\begin{aligned}
E \Pi & =\frac{1}{2}\left[\rho_{H H} \rho_{H H}\left(T_{H H}^{1}+T_{H H}^{2}\right)+2 \rho_{H H} \rho_{L H}\left(T_{H L}^{1}+T_{H L}^{2}\right)+\rho_{L H} \rho_{L H}\left(T_{L L}^{1}+T_{L L}^{2}\right)\right] \\
& +\frac{1}{2}\left[\rho_{H L} \rho_{H L}\left(T_{H H}^{1}+T_{H H}^{2}\right)+2 \rho_{H L} \rho_{L L}\left(T_{H L}^{1}+T_{H L}^{2}\right)+\rho_{L L} \rho_{L L}\left(T_{L L}^{1}+T_{L L}^{2}\right)\right]
\end{aligned}
$$

Solving (13) - (15) for $T_{H L}^{i}, T_{H H}^{i}$ and $T_{L L}^{i}$, substituting in (27) and maximizing with respect to the optimal quantities leads to

$$
\begin{aligned}
X_{H H}^{C}(\alpha, \xi) & =\frac{1}{6}\left(1+\alpha-\frac{2(1-\alpha) \xi}{1+\xi^{2}}\right) \\
X_{H L}^{C}(\alpha, \xi) & =\frac{1}{6}\left(1+\alpha-\frac{4(1-\alpha) \xi}{7-\xi^{2}}\right) \\
X_{L L}^{C}(\alpha, \xi) & =\frac{1}{6}\left(1+\alpha+\frac{60(1-\alpha)}{11\left(3+\xi^{2}\right)}-\frac{8(1-\alpha) \xi}{77-55 \xi^{2}}-\frac{6(1+\alpha) \xi}{1+\xi^{2}}\right)
\end{aligned}
$$

Using (28)-(30) shows that the remaining incentive conditions are also satisfied. More precisely, we get that

$$
\begin{aligned}
& E \Pi_{i}\left(\cdot, C_{H L}^{C}, M^{C}=\left(s_{H}, s_{L}\right)\right) \geq E \Pi_{i}\left(\cdot, C_{H H}^{C}, M^{C}=\left(s_{H}, s_{L}\right)\right) \text { and } \\
& E \Pi_{i}\left(\cdot, C_{L L}^{C}, M^{C}=\left(s_{L}, s_{L}\right)\right) \geq E \Pi_{i}\left(\cdot, C_{H L}^{C}, M^{C}=\left(s_{L}, s_{L}\right)\right), E \Pi_{i}\left(\cdot, C_{H H}^{i}, M^{C}=\left(s_{L}, s_{L}\right)\right)
\end{aligned}
$$

hold as long as $X_{H L}^{c}(\alpha, \xi) \geq 0$ which is ensured by $\alpha \geq 1 / 2$.

$X_{H H}^{C}(\alpha, \xi)$ is not distorted as $\partial T_{H L}^{i} / \partial x_{H H}^{i}=\partial T_{L L}^{i} / \partial x_{H H}^{i}=0$. The downward distortions of $X_{H L}^{C}(\alpha, \xi)$ and $X_{L L}^{C}(\alpha, \xi)$ are due to

$$
\frac{\partial T_{H H}^{i}}{\partial x_{H L}^{i}}, \frac{\partial T_{H H}^{i}}{\partial x_{L L}^{i}}<0 \text { and } \frac{\partial T_{H L}^{i}}{\partial x_{L L}^{i}}<0
$$

where all derivatives are evaluated at the equilibrium quantities.

Proof of Lemma 5 Using the optimal quantities given in lemma 1-4, the proof follows from $\alpha \in[1 / 2,1]$ and $\xi \in[0,1]$.

Proof of Corollary 1 Using the optimal quantities given in lemma 1-4, the proof 
follows from $\alpha \in[1 / 2,1]$ and $\xi \in[0,1]$, as well as some simple algebraic manipulations.

Proof of Proposition 1 The proof relies on simple but tedious comparisons of the retailers' profits under the different information sharing regimes.

Proof of Proposition 2 Calculating expected consumer surplus and adding supplier's as well as retailers' profits under the different information sharing regimes leads to the result stated in the proposition.

\subsection{Payments with complete information sharing}

The payments which ensure honest reporting of the received signals with complete information sharing are given by $z_{L L}^{C}=0$ and

$$
\begin{gathered}
z_{H H}^{C}=\frac{1}{12\left(7-5 \xi^{2}\right)^{2}\left(\xi^{2}+1\right)^{2}\left(\xi^{2}+3\right)^{2}}\left[\begin{array}{c}
3(\alpha-1)^{2} \xi^{12}+60\left(\alpha^{2}-1\right) \xi^{11}-6(\alpha-1)^{2} \xi^{10} \\
+76\left(\alpha^{2}-1\right) \xi^{9}+301(\alpha-1)^{2} \xi^{8}-344\left(\alpha^{2}-1\right) \xi^{7} \\
-756(\alpha-1)^{2} \xi^{6}+8\left(\alpha^{2}-1\right) \xi^{5}-355(\alpha-1)^{2} \xi^{4} \\
+284\left(\alpha^{2}-1\right) \xi^{3}+1914(\alpha-1)^{2} \xi^{2}-84\left(\alpha^{2}-1\right) \xi \\
-1101(\alpha-1)^{2}
\end{array}\right] \\
z_{H L}^{C}=\frac{\xi}{6\left(7-5 \xi^{2}\right)^{2}\left(\xi^{4}+4 \xi^{2}+3\right)}\left[\begin{array}{c}
3(\alpha-1)^{2} \xi^{7}+25\left(\alpha^{2}-1\right) \xi^{6}-29(\alpha-1)^{2} \xi^{5} \\
-75\left(\alpha^{2}-1\right) \xi^{4}+5(\alpha-1)^{2} \xi^{3}+111\left(\alpha^{2}-1\right) \xi^{2} \\
+37(\alpha-1)^{2} \xi-77\left(\alpha^{2}-1\right) \\
1
\end{array}\right] \\
z_{L H}^{C}=\frac{(\alpha-1)^{2} \xi^{10}+10\left(\alpha^{2}-1\right) \xi^{9}+37(\alpha-1)^{2} \xi^{8}}{12\left(\xi^{2}+1\right)\left(5 \xi^{4}+8 \xi^{2}-21\right)^{2}}\left[\begin{array}{c} 
\\
-3\left(\alpha 6\left(\alpha^{2}-1\right) \xi^{7}+462(\alpha-1)^{2} \xi^{6}+20\left(\alpha^{2}-1\right) \xi^{5}\right. \\
-562(\alpha-1)^{2} \xi^{4}-712\left(\alpha^{2}-1\right) \xi^{3}-1035(\alpha-1)^{2} \xi^{2} \\
+546\left(\alpha^{2}-1\right) v+1101(\alpha-1)^{2}
\end{array}\right]
\end{gathered}
$$

\section{References}

[1] Clarke, R. N. 1983. Collusion and the incentives for information sharing. The Bell Journal of Economics, 383-394.

[2] Dobson, P. W. and M. Waterson. 1997. Countervailing power and consumer prices. The Economic Journal 107(441), 418-430. 
[3] Ellison, S. F. and C. M. Snyder. 2010. Countervailing power in wholesale pharmaceuticals. The Journal of Industrial Economics 58(1), 32-53.

[4] Gal-Or, E. 1985. Information sharing in oligopoly. Econometrica: Journal of the Econometric Society, 329-343.

[5] Jiang, L. and Z. Hao. 2016. Incentive-Driven Information Dissemination in Two-Tier Supply Chains. Manufacturing \& Service Operations Management, 393-413.

[6] Raith, M. A. 1996. A general model of information sharing in oligopoly. Journal of Economic Theory 71(1), 260-288.

[7] Vives, X. 1984. Duopoly information equilibrium: Cournot and Bertrand. Journal of Economic Theory 34(1), 71-94.

[8] Vives, X. 1990. Trade Association Disclosure Rules, Incentives to Share Information, and Welfare. The RAND Journal of Economics 21(3), 409-430.

[9] Wagenhofer, A. 1990. Voluntary disclosure with a strategic opponent. Journal of Accounting and Economics 12(4), 341-363.

[10] Ziv, A. 1993. Information sharing in oligopoly: The truth-telling problem. The RAND Journal of Economics, 455-465. 